\title{
Gender-specific relationship between prenatal exposure to phthalates and intrauterine growth restriction
}

\author{
Yan Zhao', Li Chen', Lu-xi Li', Chang-ming Xie' ${ }^{1}$, Dan Li' ${ }^{1}$ Hui-jing Shi' and Yun-hui Zhang'
}

\begin{abstract}
BACKGROUND: No study has examined the association between prenatal phthalate exposure and intrauterine growth retardation (IUGR). This study aimed to investigate whether prenatal exposure to phthalates was associated with increased risk of IUGR.
\end{abstract}

METHODS: A total of 126 mother-newborn pairs, including 42 IUGR cases and 84 control newborns and their mothers, were enrolled in this case-control study. Spot urine samples were collected during the third trimester of pregnancy, and 5 phthalate metabolites (mono- $n$-butyl phthalate (MBP), monomethyl phthalate (MMP), mono-2-ethylhexyl phthalate (MEHP), mono(2-ethyl-5-oxohexyl) phthalate (MEOHP), and mono(2ethyl-5-hydroxyhexyl) phthalate (MEHHP)) were measured.

RESULTS: Concentrations of MMP, MEHHP, MEOHP, and SumDEHP (MEHP, MEHHP, and MEOHP) were significantly higher in IUGR cases than in normal controls. In all subjects, urinary concentrations of MEHHP and MEOHP were significantly inversely associated with fetal growth indicators (birth weight and Quetelet's index). When mothers were stratified by infant sex, MEHHP and MEOHP concentrations were still negatively associated with fetal growth indicators, while no significant association was observed in females. In addition, exposureresponse relationships were observed between MEHHP/ SumDEHP concentrations in maternal urine and IUGR.

CONCLUSION: Prenatal exposure to phthalates was associated with increased risk of IUGR, and male newborns were more sensitive to phthalates than females.

$\mathbf{P}$ hthalates, diesters of phthalic acid, are a group of chemicals that are widely used as plasticizers in many applications, including medical devices, toys, food wraps, shoe soles, and interior building surfaces (1). Because phthalates are additives and, as such, noncovalently bound to the plastic, they leach from the products with time and use, thus becoming ubiquitous environmental contaminants (2). Humans can be exposed to phthalates through inhalation, ingestion, dermal exposure, and medical treatments (3).

Once taken into the body, phthalates are metabolized to their respective monoesters within a few hours or days, and then glucuronidated and excreted in the urine and feces. A notable human burden of phthalates has been reported and the consensus is that phthalate metabolites in urine could be used for assessing phthalate exposure in humans (4-11). In most cases, it is the monoester metabolites that have been claimed to be responsible for adverse health effects $(12,13)$.

Because phthalates can penetrate the placenta in humans (14), high phthalate levels in general, and in pregnant women in particular, have raised the urgent issue of prenatal exposure to phthalates. Fetuses are specifically vulnerable to a variety of toxicants because of the immaturity of their developing immune systems and the rapid development of their organs $(15,16)$. During the fetal period, there is a "critical window" for the development of cardiovascular, nervous, reproductive, endocrine, immune, and respiratory systems when even a subtle exposure to chemicals may adversely affect morphologies and functions of the systems (17). Therefore, it is important to examine prenatal phthalate exposure and its effect on fetal health.

Toxicological evidence from animal studies demonstrated that reduced birth weight was one of the most sensitive endpoints for prenatal exposure to phthalates $(18,19)$. In female rats exposed to di- $n$-butyl phthalate (DBP, one phthalate ester), the lowest observed adverse effect level for decreased birth weight in the $\mathrm{F}_{2}$ generation was $52 \mathrm{mg} / \mathrm{kg} / \mathrm{d}$ for males and $66 \mathrm{mg} / \mathrm{kg} / \mathrm{d}$ for females (20). In human epidemiological studies (21-25), statistically significant associations have been observed between prenatal exposure to phthalates and fetal growth indicators (e.g., birth weight, birth length, and gestational age at delivery). These results together indicate that prenatal phthalate exposure may adversely affect fetal growth.

Intrauterine growth restriction (IUGR) is defined as the rate of growth of a fetus that is less than normal for the growth potential of the fetus at particular gestational age (26). Newborns with IUGR are at increased risk to develop a metabolic syndrome later in life, namely obesity, arterial hypertension, hypercholesterolemia, cardiovascular disease, impaired glucose tolerance, or diabetes mellitus type 2 (27). Regarding the etiologic factors responsible for IUGR, one-third of the variations in birth weight are determined by genetic variables, while two-thirds are determined by environmental factors (28). Although previous data showed that prenatal phthalate 
exposure was associated with reduced fetal growth, no study to date has examined the role of prenatal exposure to phthalates in the development of IUGR. Thus, we conducted this case-control study to investigate whether prenatal phthalate exposure was a risk factor for IUGR. Furthermore, considering gender differences in reproductive and developmental toxicity of phthalates, we also explored the differences in phthalate effect between males and females.

\section{RESULTS}

\section{Population Characteristics}

The maternal and pregnancy characteristics of IUGR and control patients are listed and compared as shown in Table 1. Cases and controls differed with regard to birth weight, birth length, Quetelet's index, gestational age at delivery, and passive

Table 1. Maternal and pregnancy characteristics for IUGR cases and controls

\begin{tabular}{|c|c|c|c|}
\hline Characteristics & $\begin{array}{l}\text { Cases } \\
(N=42)\end{array}$ & $\begin{array}{l}\text { Controls } \\
(N=84)\end{array}$ & $P$ value $^{c}$ \\
\hline \multicolumn{4}{|l|}{ Sex } \\
\hline Male & $20(47.6 \%)$ & $43(51.2 \%)$ & 0.705 \\
\hline Female & $22(52.4 \%)$ & $41(48.8 \%)$ & \\
\hline Birth weight $(\mathrm{kg})^{\mathrm{a}}$ & $2.43 \pm 0.36$ & $3.32 \pm 0.30$ & $0.000^{* *}$ \\
\hline Birth length $(\mathrm{cm})^{\mathrm{a}}$ & $47.90 \pm 2.26$ & $50.40 \pm 0.70$ & $0.000^{* *}$ \\
\hline Quetelet's index $\left(\mathrm{kg} / \mathrm{m}^{2}\right)^{\mathrm{a}, \mathrm{b}}$ & $10.60 \pm 1.48$ & $13.08 \pm 1.14$ & $0.000^{* *}$ \\
\hline Gestational age at delivery (wk) & $38.17 \pm 1.99$ & $39.34 \pm 1.15$ & $0.000^{* *}$ \\
\hline Prepregnant BMI $\left(\mathrm{kg} / \mathrm{m}^{2}\right)^{\mathrm{a}}$ & $19.07 \pm 2.34$ & $19.64 \pm 1.85$ & 0.067 \\
\hline $\begin{array}{l}\text { Pregnancy-induced } \\
\text { hypertension }\end{array}$ & & & 0.577 \\
\hline Yes & $5(11.9 \%)$ & $6(7.1 \%)$ & \\
\hline No & $37(88.1 \%)$ & $78(92.9 \%)$ & \\
\hline Pregnancy-induced diabetes & & & 0.748 \\
\hline Yes & $5(11.9 \%)$ & $7(8.3 \%)$ & \\
\hline No & $37(88.1 \%)$ & $77(91.7 \%)$ & \\
\hline Irregular menstruation & & & 1.000 \\
\hline Yes & $4(9.5 \%)$ & $6(7.1 \%)$ & \\
\hline No & $38(90.5 \%)$ & $78(92.9 \%)$ & \\
\hline Passive smoking & & & $0.017^{*}$ \\
\hline Yes & $27(64.3 \%)$ & $35(41.7 \%)$ & \\
\hline No & $15(35.7 \%)$ & $49(58.6 \%)$ & \\
\hline Education & & & 0.134 \\
\hline Middle school & $15(35.7 \%)$ & $17(20.2 \%)$ & \\
\hline High school & $11(26.2 \%)$ & $22(26.2 \%)$ & \\
\hline College & $16(38.1 \%)$ & $45(53.6 \%)$ & \\
\hline Monthly income per family & & & 0.306 \\
\hline$<5,000 \mathrm{rmb}$ & $20(47.6 \%)$ & $32(38.1 \%)$ & \\
\hline$\geq 5,000 \mathrm{rmb}$ & $22(52.4 \%)$ & $52(61.9 \%)$ & \\
\hline
\end{tabular}

BMI, body mass index; IUGR, intrauterine growth retardation.

aalues are shown as mean \pm SD. ${ }^{b}$ Quetelet's index was calculated as weight (in

kilograms) divided by the square of height (in meters). ${ }^{c} P$ value calculated using the $\chi^{2}$ test and the $t$-test. ${ }^{*} P<0.05,{ }^{* *} P<0.01$. smoking. Cases and controls did not differ by infant sex, maternal age, prepregnant BMI, pregnancy complications, irregular menstruation, maternal education background, and monthly income. In addition, mothers from both groups had no habit of cigarette smoking or alcohol drinking before and during pregnancy (data not shown).

\section{Urinary Phthalate Metabolites in Pregnant Women}

Urinary phthalate metabolites are considered to be good biomarkers for assessing phthalate exposure in humans because of their reliability and low contamination rate in the laboratory (29). In this study, concentrations of the five phthalate metabolites in urine samples were determined to assess prenatal phthalate exposure.

As shown in Table 2, phthalate exposure was prevalent. More than $80 \%$ of urinary samples had quantifiable levels of the five phthalate metabolites. The lower-molecular-weight phthalate metabolites mono- $n$-butyl phthalate (MBP) and monomethyl phthalate (MMP) had median values of 21.6 and $8.9 \mathrm{ng} / \mathrm{ml}$, respectively. In comparison, median concentrations of highermolecular-weight phthalate metabolites mono-2-ethylhexyl phthalate (MEHP), mono(2-ethyl-5-hydroxyhexyl) phthalate (MEHHP), and mono(2-ethyl-5-oxohexyl) phthalate (MEOHP) were 6.5, 10.6, and $4.3 \mathrm{ng} / \mathrm{ml}$, respectively.

Analyses of potential differences in phthalate metabolite concentrations in maternal urine samples between IUGR cases and controls were conducted using the Mann-Whitney U-test. Urinary concentrations of MMP, MEHHP, MEOHP, and SumDEHP were significantly higher in IUGR cases than in normal controls $(P=0.030,0.003,0.003$, and 0.004 , respectively). The largest difference in phthalate metabolites was observed in MEOHP, for which the median concentration of cases was 2.2-fold higher than in controls ( $8.0 \mathrm{ng} / \mathrm{ml}$ vs. $3.7 \mathrm{ng} / \mathrm{ml}, P=0.003$ ) (Table 2 ).

\section{Association Between Urinary Phthalate Metabolite Concentrations and Birth Outcomes}

The relationship between urinary phthalate metabolite concentrations and birth weight is shown in Figure 1. In all subjects, urinary concentrations of MMP, MEHHP, MEOHP, and SumDEHP were significantly inversely associated with birth weight. After adjusting for the potential confounders (maternal age, gestational age at delivery, maternal education, prepregnancy BMI, and passive smoking), MEHHP and MEOHP remained significant. A log-unit (10-fold) increase in urinary concentrations of MEHHP and MEOHP was associated with $0.213(\beta=-0.213 ; 95 \%$ confidence interval $(\mathrm{CI}),-0.401$ to $-0.025)$ and $0.233(\beta=-0.233$; 95\% CI, -0.411 to -0.056$) \mathrm{kg}$ decrease in birth weight, respectively. When subjects were stratified by infant gender, MEHHP ( $\beta=-0.393 ; 95 \% \mathrm{CI},-0.740$ to $-0.046)$ and MEOHP $(\beta=-0.434 ; 95 \% \mathrm{CI},-0.762$ to -0.107$)$ were still found to be inversely associated with birth weight in males even after adjusting for potential confounders. However, no phthalate metabolite was found to be significantly associated with birth weight in females.

Figure 2 presents the relationship between urinary phthalate metabolite concentrations and birth length. In the unadjusted 
Table 2. Third-trimester urinary phthalate metabolite concentrations among women who had IUGR or normal births (ng/ml)

\begin{tabular}{|c|c|c|c|c|c|c|c|c|}
\hline \multirow[b]{2}{*}{ Phthalate metabolites } & \multirow[b]{2}{*}{ LOD } & \multicolumn{3}{|c|}{$\%>$ LOD } & \multicolumn{3}{|c|}{ Median (25th, 75th percentile) } & \multirow[b]{2}{*}{$P$ value $^{\mathrm{a}}$} \\
\hline & & Total & Case & Control & Total & Case & Control & \\
\hline MBP & 0.050 & 98 & 100 & 96 & $21.6(11.0,37.6)$ & $26.5(13.0,44.7)$ & $20.0(10.1,37.3)$ & 0.199 \\
\hline MEHP & 0.050 & 83 & 95 & 76 & $6.5(1.5,17.4)$ & $8.9(3.5,16.7)$ & $5.4(0.7,17.4)$ & 0.177 \\
\hline MEHHP & 0.050 & 93 & 100 & 89 & $10.6(3.9,19.2)$ & $17.6(6.6,29.8)$ & $9.1(3.2,15.8)$ & $0.003^{* *}$ \\
\hline SumDEHPb & / & I & I & l & $26.8(13.6,46.3)$ & $37.5(16.4,54.5)$ & $20.6(9.3,41.5)$ & $0.004 * *$ \\
\hline
\end{tabular}

IUGR, intrauterine growth retardation; LOD, limits of detection; /, not applicable..

a $P$ value calculated using the Mann-Whitney- $U$-test and the $t$-test. bS SumDEHP comprises MEHP, MEHHP, and MEOHP. ${ }^{*} P<0.05$, **P $<0.01$.
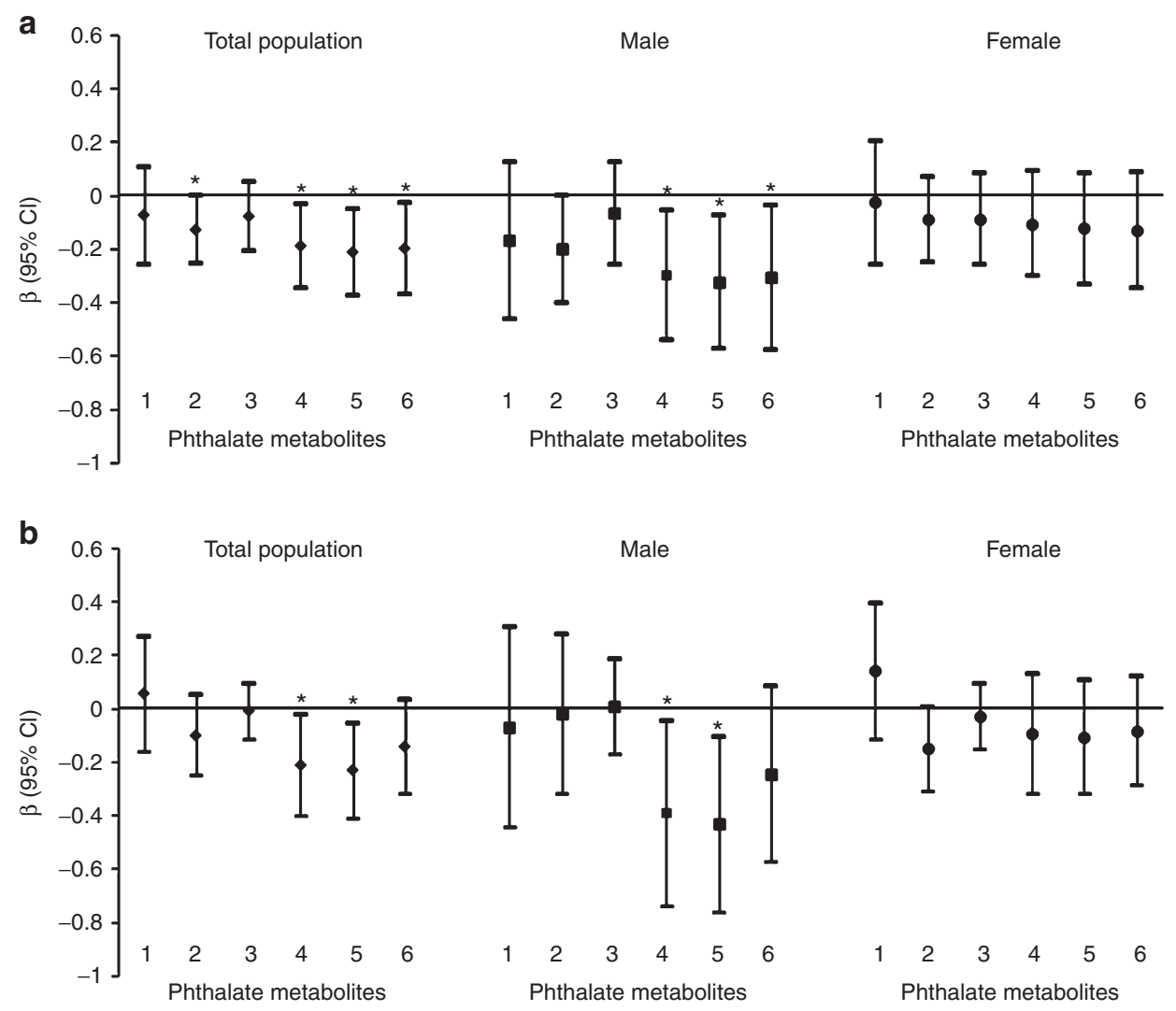

Figure 1. Associations of maternal urinary concentrations of phthalate biomarkers with birth weight. (a) Crude $\beta$ and $95 \%$ confidence intervals (Cls). (b) Adjusted $\beta$ and $95 \% \mathrm{Cls}$. Adjusted for maternal age, maternal education, gestational age, passive smoking, and other phthalate metabolites. Phthalate metabolites: 1 , mono- $n$-butyl phthalate (MBP); 2 , monomethyl phthalate (MMP); 3, mono-2-ethylhexyl phthalate (MEHP); 4, mono(2-ethyl-5-hydroxyhexyl) phthalate (MEHHP); 5, mono(2-ethyl-5-oxohexyl) phthalate (MEOHP); 6, SumDEHP. SumDEHP comprises MEHP, MEHHP, and MEOHP. *P< 0.05.

model, elevated urinary concentrations of MMP, MEHP, MEHHP, MEOHP, and SumDEHP were significantly associated with reduced birth length. Among males, urinary concentrations of higher-molecular-weight phthalate metabolites (MEHP, MEHHP, and SumDEHP) were negatively associated with birth length, while among females, it was the lower-molecular-weight phthalate metabolite (MMP) concentration that was negatively associated with birth length. However, after adjusting for potential confounders, all significances disappeared.

Figure 3 presents the regression results for phthalate metabolite levels and Quetelet's index (calculated as weight (in kilograms) divided by the square of height (in meters)). MEHHP and MEOHP concentrations were significantly associated with a decreased Quetelet's index in all subjects in both the crude and adjusted models. A log-unit (10-fold) increase in urinary concentrations of MEHHP and MEOHP was associated with a $0.656(\beta=-0.656 ; 95 \% \mathrm{CI},-1.307$ to -0.005$)$ and 0.816 $(\beta=0.816 ; 95 \% \mathrm{CI},-1.426$ to -0.206$) \mathrm{kg} / \mathrm{m}^{2}$ decrease in birth weight, respectively. There was an obvious gender difference in the associations between MEHHP/MEOHP concentration and Quetelet's index, which was stronger in males and not statistically significant in females.

Although IUGR newborns seemed to be having shorter gestational age than control newborns $(38.17 \mathrm{wk}$ vs. 

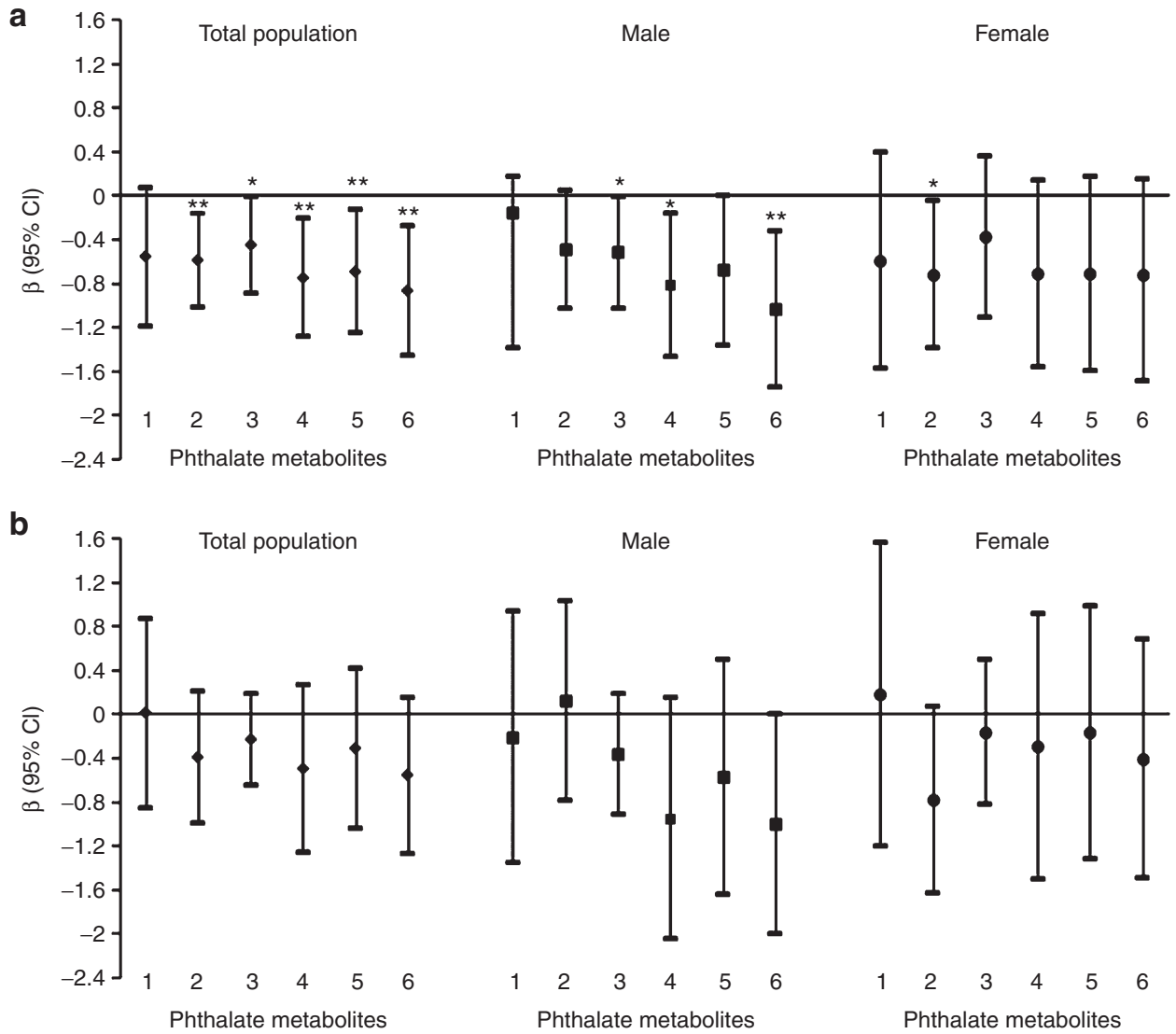

Figure 2. Associations of maternal urinary concentrations of phthalate biomarkers with birth length. (a) Crude $\beta$ and $95 \%$ confidence intervals (Cls). (b) Adjusted $\beta$ and $95 \%$ Cls. Adjusted for maternal age, maternal education, gestational age, passive smoking, and other phthalate metabolites. Phthalate metabolites: 1, mono- $n$-butyl phthalate (MBP); 2, monomethyl phthalate (MMP); 3, mono-2-ethylhexyl phthalate (MEHP); 4, mono(2-ethyl-5-hydroxyhexyl) phthalate (MEHHP); 5, mono(2-ethyl-5-oxohexyl) phthalate (MEOHP); 6, SumDEHP. SumDEHP comprises MEHP, MEHHP, and MEOHP. *P $<0.05, * * P<0.01$.

39.34 wk; $P=0.000$ ), we did not find any association between urinary phthalate metabolite concentrations and gestational age (data not shown).

\section{Exposure-Response Relationships of Prenatal Phthalate Exposure and IUGR in Newborns}

As shown in Figure 4, MEHHP (OR=4.68; 95\%CI, 1.76 to 12.45), MEOHP (OR=3.41; 95\%CI, 1.36 to 8.54), and SumDEHP (odds ratio $(\mathrm{OR})=3.64 ; 95 \% \mathrm{CI}, 1.04$ to 6.73 ) concentrations in the highest tertile were significantly associated with an increased risk of IUGR. Higher concentrations of MBP, MMP, MEHHP, and SumDEHP were associated with the risk of IUGR in an exposure-response relationship manner. However, after adjusting for gestational age at delivery, infant gender, maternal education, and passive smoking, only MEHHP (OR=5.80; 95\%CI, 1.55 to 21.67$)$ concentration in the highest tertile remained statistically significant. Exposure-response relationships were observed between MEHHP/SumDEHP concentration in maternal urine and IUGR, shown by increasing ORs.

\section{DISCUSSION}

As commonly used plasticizers, phthalates are ubiquitous in an environment matrix and human bodies. Studies have reported quantifiable levels of phthalates in pregnant women
(30-32), which indicated that prenatal phthalate exposure is common. As prenatal phthalate exposure has been shown to be associated with adverse fetal growth, we sought to investigate the association between prenatal exposure to phthalates and IUGR.

A single urine sample could be moderately predictive of each subject's exposure over 3 mo $(22,29)$. Therefore, urinary phthalate metabolite concentrations during the third trimester of pregnancy could be used for estimating prenatal exposure to phthalates. In this study, the detection rate of phthalate metabolites was over $80 \%$, which indicated that exposure to phthalates was a routine occurrence for the present subjects. The median concentrations of phthalate metabolites ranged from 4.3 to $21.6 \mathrm{ng} / \mathrm{ml}$ (Table 2). These levels were generally comparable with those reported in urine samples collected from pregnant women in Germany (33), Israel (34), Japan (17), Norway (32), Peru (35), Spain (36), Sweden (6), and the United States (30), but the concentrations of DEHP metabolites in our study were somewhat lower.

Our finding that urinary concentrations of phthalate metabolites were negatively associated with fetal growth indicators is consistent with our own previous study (24). In that study, we determined phthalate levels in umbilical cord blood and fetal meconium, and found that DBP exposure in utero (DBP in 

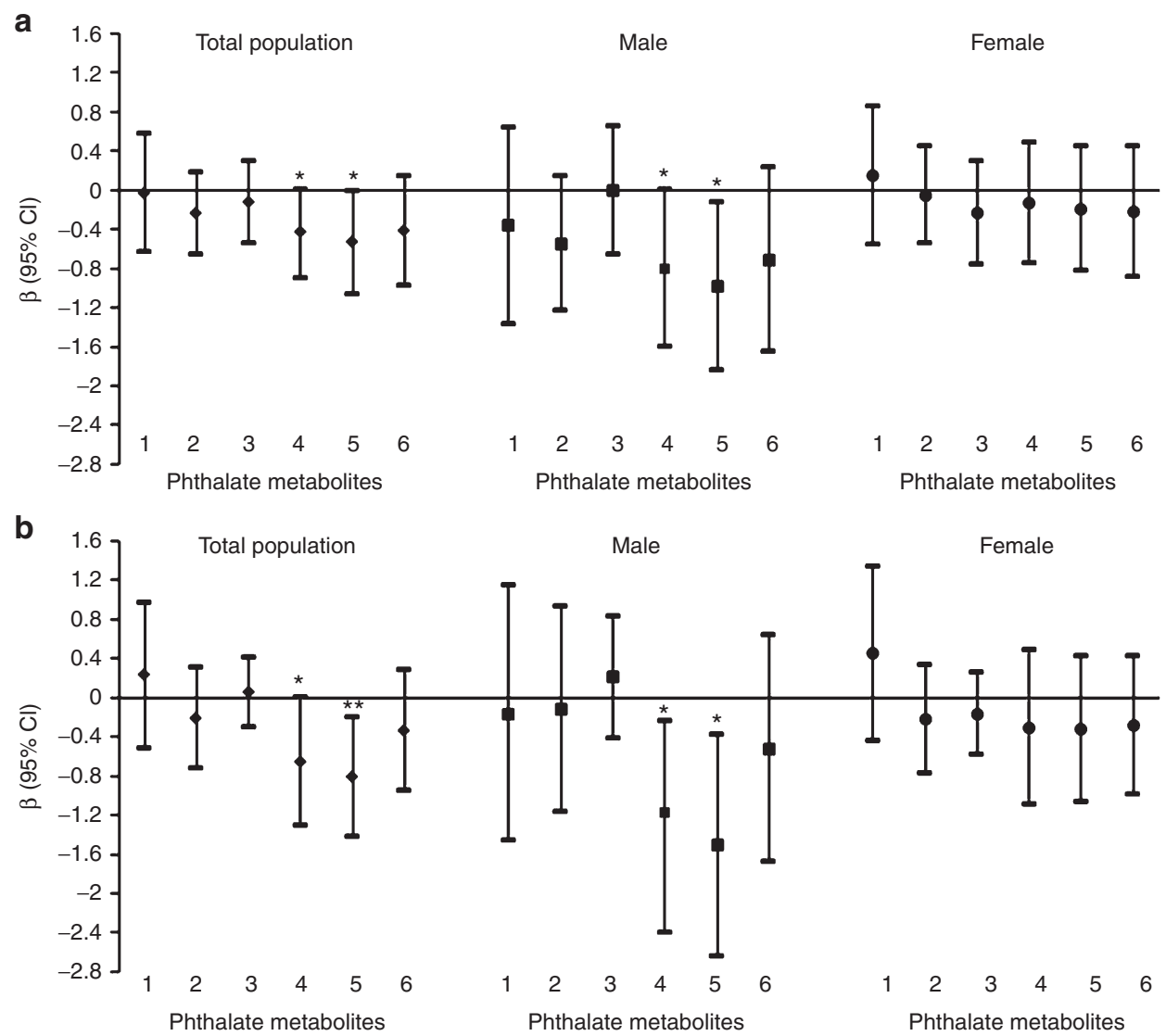

Figure 3. Associations of maternal urinary concentrations of phthalate biomarkers with Quetelet's index. (a) Crude $\beta$ and $95 \%$ confidence intervals (Cls). (b) Adjusted $\beta$ and $95 \% \mathrm{Cls}$. Adjusted for maternal age, maternal education, gestational age, passive smoking, and other phthalate metabolites. Phthalate metabolites: 1, mono-n-butyl phthalate (MBP); 2, monomethyl phthalate (MMP); 3, mono-2-ethylhexyl phthalate (MEHP); 4, mono(2-ethyl-5-hydroxyhexyl) phthalate (MEHHP); 5, mono(2-ethyl-5-oxohexyl) phthalate (MEOHP); 6, SumDEHP. SumDEHP comprises MEHP, MEHHP, and MEOHP. ${ }^{*} P<0.05,{ }^{* *} P<0.01$.

cord blood and MBP in meconium) was negatively associated with birth weight, and DEHP exposure (MEHP in cord blood and meconium) was associated with shorter birth length. Especially in this present study, we found a notable difference in effect between males and females. Among males, maternal urinary concentrations of higher-molecular-weight phthalate metabolites (MEHHP and MEOHP) were significantly inversely associated with birth weight and Quetelet's index, while among females no significant association was observed. Our results suggest that male fetuses are more sensitive to prenatal phthalate exposure than females.

Previous studies on association between prenatal phthalate exposure and gestational age yielded inconsistent results. Meeker et al. (25) reported that mothers of preterm birth infants had significantly higher urinary MBP and monobenzyl phthalate (MBzP) concentrations during pregnancy than those of full-term infants. In contrast, Wolff et al. (31) found that lower-molecular-weight phthalate metabolites in the urine of pregnant women had a positive association with gestational age. However, we did not find any association between urinary phthalate metabolite concentrations and gestational age, which was consistent with the results of two recent studies $(17,37)$. These divergent results might be attributed to diversity between the levels of phthalates measured in different studies.
The most remarkable result in this study is the exposureresponse relationship between urinary phthalate metabolite concentrations and increased IUGR risk. ORs for IUGR were found to be increased with the increasing of MEHHP and SumDEHP exposure levels, and exposure-response relationships were observed between urinary phthalate metabolite concentrations and IUGR risk, especially in the highest tertile (OR up to 5.80). These results indicate that fetuses might be at a higher risk for IUGR after prenatal exposure to phthalates.

There are some limitations in our study. Firstly, our sample size is relatively small. Therefore, statistical power to detect subtle changes might be low. Secondly, phthalate metabolites have short half-lives and our exposure measurements relied on a single urine specimen. Misclassification of exposure and outcome in our study is possible, although they are likely to be independent of one another. Thus, some other biomarkers might be needed to assess prenatal phthalate exposure. Furthermore, the potential confounding from exposure to other pollutants is unclear and cannot be excluded.

In conclusion, the present study provides evidence of potential roles of prenatal phthalate exposure in the development of IUGR among a group of Chinese women. The detection of these associations, even in this small sample size study, may have significant implications due to the widespread distribution 

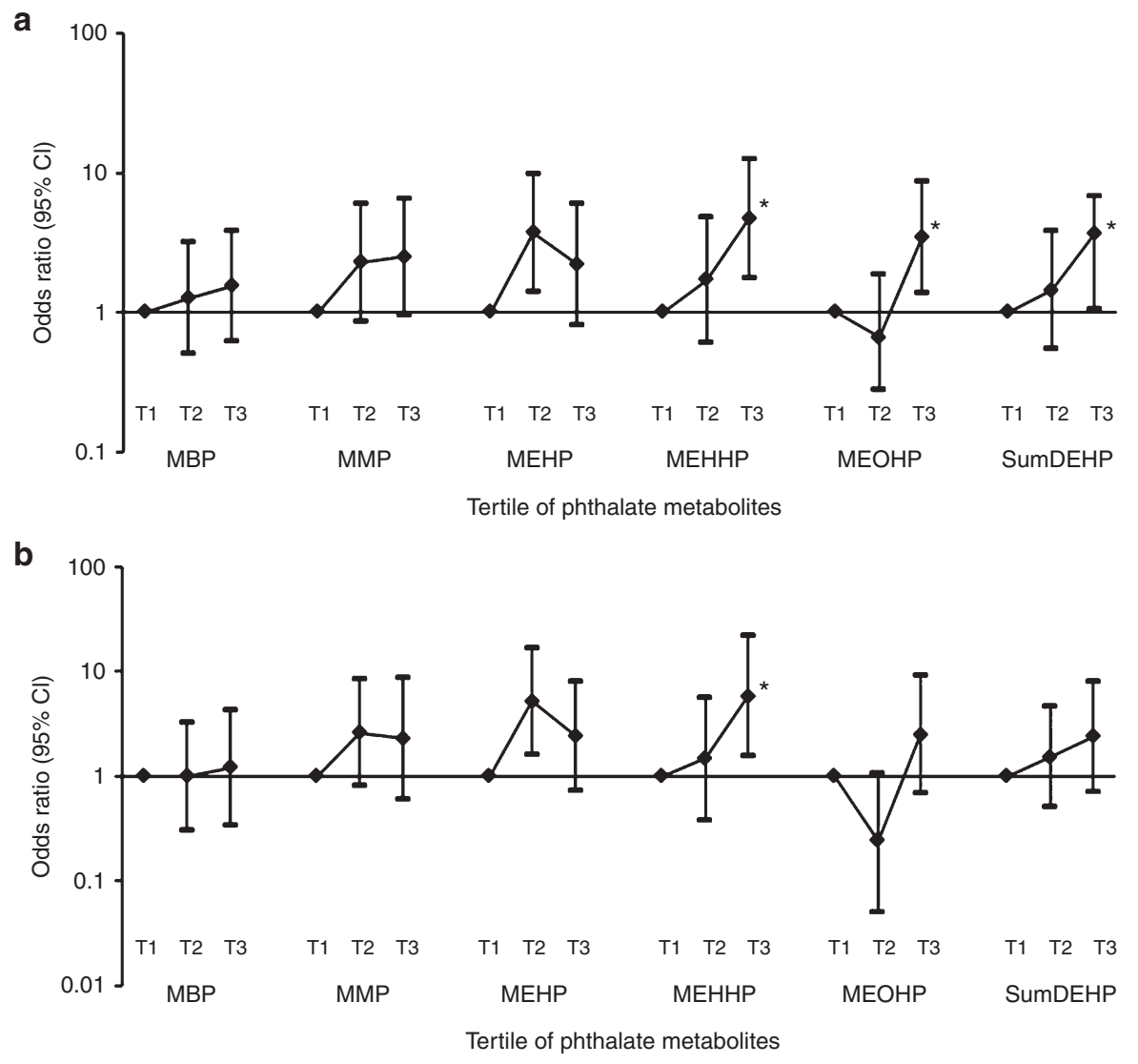

Figure 4. Odds ratios (ORs) and $95 \%$ confidence intervals $(\mathrm{Cls})$ for the associations between phthalate metabolite concentrations in maternal urine and intrauterine growth retardation (IUGR). (a) Crude model. (b) Adjusted model. Adjusted for gestational age, infant sex, passive smoking, and other phthalate metabolites (in tertiles). Tertile of phthalate metabolites: $\mathrm{T1}, 0-33.3$ th percentile; $T 2,33.3-66.7$ th percentile; T3, 66.7th percentile-maximum value. SumDEHP comprises mono-2-ethylhexyl phthalate (MEHP), mono(2-ethyl-5-hydroxyhexyl) phthalate (MEHHP), and mono(2-ethyl-5-oxohexyl) phthalate (MEOHP). ${ }^{*} P<0.05$.

of phthalates in the general population. Further studies with a larger sample size, and with more known confounders taken into account, are needed to investigate the causality in these relationships.

\section{METHODS}

\section{Study Population}

Participants were recruited during their third-trimester examination at the Second Affiliated Hospital of Wenzhou Medical College between March 2012 and January 2013. IUGR was defined as estimated fetal weight (EFW) below the 10th centile for gestational age, based on sonographic measurements of fetal biparietal diameter, head circumference, abdominal circumference, and femur length. For each IUGR fetus, two healthy fetuses were selected as controls matched for maternal age. During the study period, 126 mother-newborn pairs, including 42 IUGR cases and 84 controls and their mothers, were enrolled in our study. All subjects were residents of Wenzhou and signed written informed consents approved by Fudan University's Institutional Review Board. In $2 \mathrm{~d}$ after delivery, the mothers answered a detailed questionnaire concerning maternal weight and height before pregnancy, education background, dietary habits, monthly income, and so on. The demographic characteristics of newborns, including birth weight, birth length, and gestational age (calculated by subtracting the date of the last menstrual period from the date of delivery), were obtained from hospital records.

\section{Sample Collection and Measurement}

Maternal urine samples were obtained during the third trimester. All specimens were collected with glass devices to avoid contamination by phthalates during handling and storage. Urine samples were stored at $-20^{\circ} \mathrm{C}$ until analysis.

Five phthalate metabolites measured here included two lowermolecular-weight phthalate metabolites (MBP and MMP) and three higher-molecular-weight phthalate metabolites (MEHP, MEOHP, and MEHHP). MBP and MMP are primary metabolites of DBP and dimethyl phthalate (DMP), respectively. MEHP is the primary metabolite of DEHP, and MEHHP and MEOHP are secondary metabolites of DEHP.

Urinary phthalate metabolites were analyzed using a method modified by Guo et al. (38). Briefly, $0.5 \mathrm{ml}$ of urine sample was enzymatically hydrolyzed and purified by solid-phase extraction. Phthalate metabolites in urine extract were resolved by an Agilent 1100 Series high-performance liquid chromatography (HPLC) system (Agilent Technologies, Santa Clara, CA) and detected by an API 2000 electrospray triple quadrupole mass spectrometer (ESI-MS/MS; Applied Biosystems, Foster City, CA). The limits of detection (LOD) were in the low nanogram per milliliter range for each phthalate metabolite. ${ }^{13} \mathrm{C}_{4}$-labeled internal standards and conjugated internal standards were used to increase the precision of the measurements. One method blank, two quality control samples, and two sets of standards were analyzed along with unknown urine samples. Analysts at the Key Laboratory of Public Health Safety, Ministry of Education were blind to all information concerning our subjects.

Due to the glucuronidation of phthalate metabolites in the liver and their elimination by active tubular secretion, creatinine correction may not be appropriate for urinary phthalate metabolite concentration $(29,39)$. Thus, in this study, specific gravity was used to correct for urinary dilution, as recommended by Hauser et al. (29). Specific gravity was measured using a handheld refractometer (Atago PAL 
10-S, Tokyo, Japan). The correction formula was $P=P \times[(1.024-1) /$ (SG-1)], where $P$ is the specific-gravity-corrected phthalate metabolite concentration $(\mathrm{ng} / \mathrm{ml}), P$ is the experimental phthalate metabolite concentration $(\mathrm{ng} / \mathrm{ml})$, and SG is the specific gravity of the urine sample (40).

\section{Data Analysis}

We performed the statistical analyses using the SPSS 16.0 statistical package (SPSS, Chicago, IL). Maternal and pregnancy characteristics were tabulated and compared between IUGR cases and controls using parametric $t$-tests and $\chi^{2}$ tests when appropriate. For phthalate metabolite concentrations below the LOD, we used an imputed value equal to one-half the LOD. In addition to the five individual phthalate metabolites, we calculated the concentrations of total DEHP metabolites (SumDEHP) by summing the concentrations of three individual DEHP metabolites: MEHP, MEHHP, and MEOHP. As urinary phthalate metabolite concentrations were not normally distributed, medians were presented to characterize urinary phthalate metabolite concentrations in the descriptive analysis. Analyses of potential differences in urinary phthalate metabolite concentrations between IUGR cases and normal controls were conducted using the Mann-Whitney $U$-test.

Multiple linear regression models were used to model the associations of $\log 10$-transformed phthalate metabolite concentrations and fetal growth indicators (birth weight, birth length, and Quetelet's index). Covariates were included in multivariate analyses if they differed significantly between cases and controls at a 0.20 level of significance. The included covariates in our model were maternal age (a matching factor), gestational age at delivery, maternal education, prepregnancy BMI, and passive smoking. In addition, because urinary phthalate metabolite concentrations were correlated with each other, especially between metabolites derived from one parent phthalate (Supplementary Table S1 online), urine phthalate metabolite concentrations were also included as covariates. In order to avoid the collinearity derived from strong correlations among daughter metabolites of one parent phthalate in the linear regression model, only one daughter metabolite was included in the adjusted model. Furthermore, the distribution of each phthalate metabolite was divided into tertiles, and an OR was calculated for each tertile compared with the lowest tertile. Conditional logistic regression was used to examine the relationship between each phthalate metabolite and IUGR, taking into account gestational age at delivery, infant sex, passive smoking, maternal education, and other phthalate metabolite variables. The analyses were considered statistically significant when $P<0.05$.

\section{SUPPLEMENTARY MATERIAL}

Supplementary material is linked to the online version of the paper at http://www.nature.com/pr

\section{STATEMENT OF FINANCIAL SUPPORT}

This study was supported by the Natural Science Foundation of China (grant 81072263 to Y.Z.; grant 81172684 to H.S.), the Shanghai Natural Science Foundation (grant 10ZR1402000 to Y.Z.), and the Shanghai Municipal Health Bureau (grant 12GWZX0301 to H.S.).

Disclosure: The authors declare no conflict of interest.

\section{REFERENCES}

1. Schettler T. Human exposure to phthalates via consumer products. Int J Androl 2006;29:134-9; discussion 181-5.

2. Heudorf U, Mersch-Sundermann V, Angerer J. Phthalates: toxicology and exposure. Int J Hyg Environ Health 2007;210:623-34.

3. Latini G. Monitoring phthalate exposure in humans. Clin Chim Acta 2005;361:20-9.

4. Silva MJ, Barr DB, Reidy JA, et al. Urinary levels of seven phthalate metabolites in the U.S. population from the National Health and Nutrition Examination Survey (NHANES) 1999-2000. Environ Health Perspect 2004;112:331-8.

5. Koch HM, Rossbach B, Drexler H, Angerer J. Internal exposure of the general population to DEHP and other phthalates-determination of secondary and primary phthalate monoester metabolites in urine. Environ Res 2003;93:177-85.

6. Högberg J, Hanberg A, Berglund M, et al. Phthalate diesters and their metabolites in human breast milk, blood or serum, and urine as biomarkers of exposure in vulnerable populations. Environ Health Perspect 2008;116:334-9.

7. López-Carrillo L, Hernández-Ramírez RU, Calafat AM, et al. Exposure to phthalates and breast cancer risk in northern Mexico. Environ Health Perspect 2010;118:539-44.

8. Itoh H, Yoshida K, Masunaga S. Quantitative identification of unknown exposure pathways of phthalates based on measuring their metabolites in human urine. Environ Sci Technol 2007;41:4542-7.

9. Itoh $H$, Iwasaki $M$, Hanaoka T, Sasaki H, Tanaka T, Tsugane S. Urinary phthalate monoesters and endometriosis in infertile Japanese women. Sci Total Environ 2009;408:37-42.

10. Ji K, Lim Kho Y, Park Y, Choi K. Influence of a five-day vegetarian diet on urinary levels of antibiotics and phthalate metabolites: a pilot study with "Temple Stay" participants. Environ Res 2010;110:375-82.

11. Guo Y, Wu Q, Kannan K. Phthalate metabolites in urine from China, and implications for human exposures. Environ Int 2011;37:893-8.

12. Frederiksen H, Skakkebaek NE, Andersson AM. Metabolism of phthalates in humans. Mol Nutr Food Res 2007;51:899-911.

13. Wittassek M, Angerer J. Phthalates: metabolism and exposure. Int J Androl 2008;31:131-8.

14. Mose T, Mortensen GK, Hedegaard M, Knudsen LE. Phthalate monoesters in perfusate from a dual placenta perfusion system, the placenta tissue and umbilical cord blood. Reprod Toxicol 2007;23:83-91.

15. Perera FP, Jedrychowski W, Rauh V, Whyatt RM. Molecular epidemiologic research on the effects of environmental pollutants on the fetus. Environ Health Perspect 1999;107:Suppl 3:451-60.

16. Srám R. Impact of air pollution on reproductive health. Environ Health Perspect 1999;107:A542-3.

17. Suzuki Y, Niwa M, Yoshinaga J, Mizumoto Y, Serizawa S, Shiraishi H. Prenatal exposure to phthalate esters and PAHs and birth outcomes. Environ Int 2010;36:699-704.

18. Tanaka T. Reproductive and neurobehavioural toxicity study of bis(2-ethylhexyl) phthalate (DEHP) administered to mice in the diet. Food Chem Toxicol 2002;40:1499-506.

19. Tyl RW, Myers CB, Marr MC, et al. Reproductive toxicity evaluation of dietary butyl benzyl phthalate (BBP) in rats. Reprod Toxicol 2004;18:241-64.

20. Marsman D. NTP technical report on the toxicity studies of dibutyl phthalate (CAS No. 84-74-2) administered in feed to $\mathrm{F} 344 / \mathrm{N}$ rats and B6C3F1 mice. Toxic Rep Ser 1995;30:1-G5.

21. Latini G, De Felice C, Presta G, et al. In utero exposure to di-(2-ethylhexyl) phthalate and duration of human pregnancy. Environ Health Perspect 2003;111:1783-5.

22. Teitelbaum SL, Britton JA, Calafat AM, et al. Temporal variability in urinary concentrations of phthalate metabolites, phytoestrogens and phenols among minority children in the United States. Environ Res 2008;106: 257-69.

23. Adibi JJ, Hauser R, Williams PL, et al. Maternal urinary metabolites of di-(2-ethylhexyl) phthalate in relation to the timing of labor in a US multicenter pregnancy cohort study. Am J Epidemiol 2009;169:1015-24.

24. Zhang Y, Lin L, Cao Y, Chen B, Zheng L, Ge RS. Phthalate levels and low birth weight: a nested case-control study of Chinese newborns. J Pediatr 2009;155:500-4.

25. Meeker JD, Hu H, Cantonwine DE, et al. Urinary phthalate metabolites in relation to preterm birth in Mexico city. Environ Health Perspect 2009;117:1587-92.

26. Longo S, Bollani L, Decembrino L, Di Comite A, Angelini M, Stronati M. Short-term and long-term sequelae in intrauterine growth retardation (IUGR). J Matern Fetal Neonatal Med 2013;26:222-5.

27. Kanaka-Gantenbein C, Mastorakos G, Chrousos GP. Endocrine-related causes and consequences of intrauterine growth retardation. Ann NY Acad Sci 2003;997:150-7.

28. Lepercq J, Mahieu-Caputo D. Diagnosis and management of intrauterine growth retardation. Horm Res 1998;49:Suppl 2:14-9. 
29. Hauser R, Meeker JD, Park S, Silva MJ, Calafat AM. Temporal variability of urinary phthalate metabolite levels in men of reproductive age. Environ Health Perspect 2004;112:1734-40.

30. Adibi JJ, Whyatt RM, Williams PL, et al. Characterization of phthalate exposure among pregnant women assessed by repeat air and urine samples. Environ Health Perspect 2008;116:467-73.

31. Wolff MS, Engel SM, Berkowitz GS, et al. Prenatal phenol and phthalate exposures and birth outcomes. Environ Health Perspect 2008;116:1092-7.

32. Ye X, Pierik FH, Angerer J, et al. Levels of metabolites of organophosphate pesticides, phthalates, and bisphenol $\mathrm{A}$ in pooled urine specimens from pregnant women participating in the Norwegian Mother and Child Cohort Study (MoBa). Int J Hyg Environ Health 2009;212:481-91.

33. Kasper-Sonnenberg M, Koch HM, Wittsiepe J, Wilhelm M. Levels of phthalate metabolites in urine among mother-child-pairs-results from the Duisburg birth cohort study, Germany. Int J Hyg Environ Health 2012;215: 373-82.

34. Berman T, Hochner-Celnikier D, Calafat AM, et al. Phthalate exposure among pregnant women in Jerusalem, Israel: results of a pilot study. Environ Int 2009;35:353-7.
35. Irvin EA, Calafat AM, Silva MJ, et al. An estimate of phthalate exposure among pregnant women living in Trujillo, Peru. Chemosphere 2010;80:1301-7.

36. Casas L, Fernandez MF, Llop S, et al. Urinary concentrations of phthalates and phenols in a population of Spanish pregnant women and children. Environ Int 2011;37:858-66.

37. Huang PC, Kuo PL, Chou YY, Lin SJ, Lee CC. Association between prenatal exposure to phthalates and the health of newborns. Environ Int 2009;35:14-20.

38. Guo Y, Alomirah H, Cho HS, et al. Occurrence of phthalate metabolites in human urine from several Asian countries. Environ Sci Technol 2011;45:3138-44.

39. Hines EP, Calafat AM, Silva MJ, Mendola P, Fenton SE. Concentrations of phthalate metabolites in milk, urine, saliva, and serum of lactating North Carolina women. Environ Health Perspect 2009;117: $86-92$.

40. Boeniger MF, Lowry LK, Rosenberg J. Interpretation of urine results used to assess chemical exposure with emphasis on creatinine adjustments: a review. Am Ind Hyg Assoc J 1993;54:615-27. 